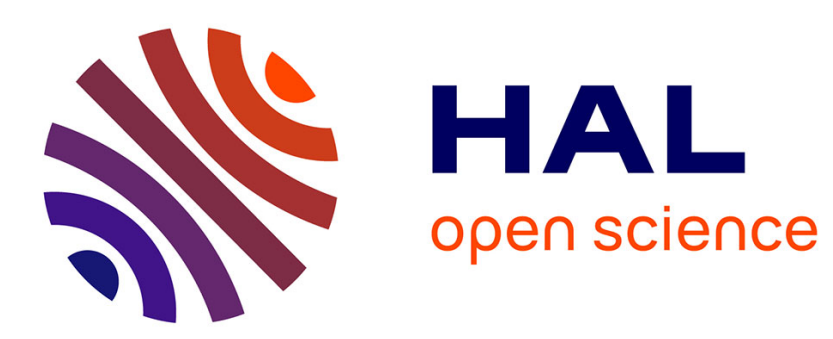

\title{
Dispersive effect of cross-aging with archimedean copulas
}

\author{
Michel M. Denuit, Mhamed Mesfioui
}

\section{To cite this version:}

Michel M. Denuit, Mhamed Mesfioui. Dispersive effect of cross-aging with archimedean copulas. Statistics and Probability Letters, 2011, 81 (9), pp.1407. 10.1016/j.spl.2011.04.011 . hal-00762902

\section{HAL Id: hal-00762902 \\ https://hal.science/hal-00762902}

Submitted on 9 Dec 2012

HAL is a multi-disciplinary open access archive for the deposit and dissemination of scientific research documents, whether they are published or not. The documents may come from teaching and research institutions in France or abroad, or from public or private research centers.
L'archive ouverte pluridisciplinaire HAL, est destinée au dépôt et à la diffusion de documents scientifiques de niveau recherche, publiés ou non, émanant des établissements d'enseignement et de recherche français ou étrangers, des laboratoires publics ou privés. 


\section{Accepted Manuscript}

Dispersive effect of cross-aging with archimedean copulas

Michel M. Denuit, Mhamed Mesfioui

PII:

S0167-7152(11)00150-7

DOI:

10.1016/j.spl.2011.04.011

Reference: $\quad$ STAPRO 5986

To appear in: Statistics and Probability Letters

Received date: 22 August 2010

Revised date: 15 February 2011

Accepted date: 17 April 2011

Please cite this article as: Denuit, M.M., Mesfioui, M., Dispersive effect of cross-aging with archimedean copulas. Statistics and Probability Letters (2011), doi:10.1016/j.spl.2011.04.011

This is a PDF file of an unedited manuscript that has been accepted for publication. As a service to our customers we are providing this early version of the manuscript. The manuscript will undergo copyediting, typesetting, and review of the resulting proof before it is published in its final form. Please note that during the production process errors may be discovered which could affect the content, and all legal disclaimers that apply to the journal pertain. 


\title{
DISPERSIVE EFFECT OF CROSS-AGING WITH ARCHIMEDEAN COPULAS
}

\author{
MiCHEL M. DENUIT \\ Institut de statistique, biostatistique et sciences actuarielles (ISBA) \\ Université Catholique de Louvain \\ B-1348 Louvain-la-Neuve, Belgium \\ MHAMED MESFIOUI \\ Département de mathématiques et d'informatique \\ Université du Québec à Trois-Rivières \\ Trois-Rivières (Québec) \\ Canada G9A 5H7
}

February 15, 2011 


\begin{abstract}
In this paper, we compare conditional distributions derived from bivariate archimedean copulas in terms of their respective variability using the dispersive stochastic order. Specifically, we fix the underlying copula and we consider the effect of increasing the second component on the variability of the conditional distribution of the first component. Characterizations are provided in terms of the generator and of the marginal distributions. Several examples involving standard parametric copulas such as Clayton and Frank are discussed.
\end{abstract}

Key words and phrases: copula, dependence, dispersive order.

Classification: 60E15 (Inequalities; stochastic orderings) 


\section{Introduction and motivation}

In this paper, we consider random couples $\left(X_{1}, X_{2}\right)$ with archimedean copula generated by a continuous, possibly infinite, strictly decreasing convex function $\phi:[0,1] \rightarrow \mathbb{R}^{+}$such that $\phi(1)=0$. Specifically, define the pseudo-inverse $\phi^{[-1]}$ of the generator $\phi$ as

$$
\phi^{[-1]}(t)=\left\{\begin{array}{l}
\phi^{-1}(t) \text { for } 0 \leq t \leq \phi(0) \\
0 \text { for } \phi(0) \leq t \leq+\infty
\end{array}\right.
$$

Clearly, $\phi^{[-1]}$ is continuous and non-increasing on $\mathbb{R}^{+}$, and strictly decreasing on $[0, \phi(0)]$. For a strict generator (i.e. a generator $\phi$ such that $\left.\lim _{t \rightarrow 0} \phi(t)=+\infty\right), \phi^{[-1]}$ is just the inverse $\phi^{-1}$ of $\phi$. For a non-strict generator (i.e. a generator $\phi$ such that $\left.\phi(0)<+\infty\right)$, $\phi^{[-1]}$ coincides with the inverse $\phi^{-1}$ of $\phi$ on $[0, \phi(0)]$ and is set equal to 0 after $\phi(0)$. Now, we consider random couples $\left(X_{1}, X_{2}\right)$ with joint distribution function $F_{\boldsymbol{X}}$ of the form

$$
F_{\boldsymbol{X}}\left(x_{1}, x_{2}\right)=C_{\phi}\left(F_{1}\left(x_{1}\right), F_{2}\left(x_{2}\right)\right)
$$

where for $t \in \mathbb{R}, F_{i}(t)=\operatorname{Pr}\left[X_{i} \leq t\right], i=1,2$, and where $C_{\phi}$ is the archimedean copula with generator $\phi$ defined as

$$
C_{\phi}\left(u_{1}, u_{2}\right)=\phi^{[-1]}\left(\phi\left(u_{1}\right)+\phi\left(u_{2}\right)\right)
$$

for $0 \leq u_{1}, u_{2} \leq 1$. Throughout the paper, we assume that the marginal distribution functions $F_{1}$ and $F_{2}$ are continuous and strictly increasing on their support. If needed, we also assume that $F_{1}$ and $F_{2}$ possess probability density functions, denoted as $f_{1}$ and $f_{2}$, respectively.

Archimedean copulas (1.3) enjoy numerous convenient mathematical properties and are therefore appreciated for modelling or simulating bivariate data. See, e.g., Nelsen (2006, Chapter 4) for a review. In particular, archimedean copulas naturally appear in relation with frailty models for the joint distribution of two survival times depending on the same latent factor (the generator being then the inverse of the Laplace transform of this latent factor).

Here, we examine the behavior of one component of a random vector when the other component gets larger, or ages. Specifically, we compare the variability of $X_{1}$ given $X_{2}=x_{2}$ to the variability of $X_{1}$ given $X_{2}=x_{2}^{\prime}$ with $x_{2}<x_{2}^{\prime}$ in terms of the dispersive order. Note that the copula $C_{\phi}$ is held fixed in our analysis so that we do not modify the underlying dependence structure but we study the impact of the aging of $X_{2}$ on the variability of $X_{1}$. We refer to this phenomenon as cross-aging.

Random couples with joint distribution function (1.2) often possess positive dependence properties, ensuring that $X_{1}$ "increases" in $X_{2}$, and vice versa. Formally, recall the definition of stochastic dominance, which translates into mathematical terms the intuitive idea of "being larger than" for random variables: given the random variables $X$ and $Y, X$ is said to be smaller than $Y$ in the stochastic dominance (denoted as $X \preceq_{\text {st }} Y$ ) if $\operatorname{Pr}[X \leq t] \geq$ $\operatorname{Pr}[Y \leq t]$ for all $t$. Whatever the threshold $t$, it is thus more likely that $X$ falls below $t$ compared to $Y$. Given a random variable $X$ and an event $A$, let us denote as $[X \mid A]$ a random variable with distribution function $x \mapsto \operatorname{Pr}[X \leq x \mid A]$. If the generator $\phi$ is differentiable and if its derivative is log-concave, Muller and Scarsini (2005, Theorem 2.8) established that 
$\left[X_{1} \mid X_{2}=x_{2}\right] \preceq_{\text {st }}\left[X_{1} \mid X_{2}=x_{2}^{\prime}\right]$ for $x_{2} \leq x_{2}^{\prime}$ when $\left(X_{1}, X_{2}\right)$ has distribution function (1.2). This property is referred to as conditional increasingness in the literature. If $C_{\phi}$ is obtained from the frailty construction (i.e. the generator $\phi$ is the inverse of a Laplace transform) then the above stochastic inequality holds with the stronger likelihood ratio order replacing $\preceq_{\text {st }}$. This property is known in the literature as total positivity of degree 2 (TP2) and is fulfilled by most parametric families of archimedean copulas. For more results in that direction, we refer the interested readers, e.g., to Denuit et al. (2005, Chapter 5).

Whereas $X_{1}$ generally "increases" in $X_{2}$, a natural question that has to the best of our knowledge not yet been addressed in the literature concerns the variability of $X_{1}$ given $X_{2}$. When $X_{2}$ is known to increase, does $X_{1}$ become more or less variable? In this paper, we answer this question using the dispersive order which turns out to be the appropriate tool to study the variability of conditionals derived from archimedean copulas provided some conditions are met. The dispersive order is one of the strongest variability orders, making the results derived in the present paper particularly attractive for applications. Note that the usual convex order does not apply in our case since the conditional mean is generally not constant.

The paper proceeds as follows. Section 2 recalls basic facts about dispersive order. In Section 3, we examine the case of unit uniform marginals. The strictness of the generators turns out to play an important role in the analysis conducted there. Then, in Section 4, we allow for arbitrary marginals. This general case is not a direct consequence of the preceding section as the marginal behavior does matter. In Section 5, we establish comparative results where cross-aging (in the dispersive sense) provides the appropriate theoretical argument. The final Section 6 concludes.

\section{Dispersive order}

The dispersive order can be used for comparing spread among probability distributions. Considering two random variables $X$ and $Y, X$ is smaller than $Y$ in the dispersive order when the difference between any two quantiles of $X$ is smaller than the difference between the corresponding quantiles of $Y$. The dispersive order has a long history in statistics. We refer the reader e.g. to the review paper by Jeon et al. (2006) as well as to the reference book by Shaked and Shanthikumar (2007) for a detailed presentation of this stochastic order relation. In the context of lifetime distributions, it has been used by Belzunce et al. (1996) and Pellerey and Shaked (1997) to characterize IFR and DFR distributions.

Define the generalized inverse (or quantile function) of the distribution function $F$ for $\alpha \in(0,1)$ by

$$
F^{-1}(\alpha)=\inf \{x \in \mathbb{R} \mid F(x) \geq \alpha\} .
$$

Furthermore, $F^{-1}(0)$ is defined as the left endpoint of the support of $F$, i.e. $F^{-1}(0)=$ $\sup \{x \in \mathbb{R} \mid F(x)=0\}$, with the convention $\sup \emptyset=-\infty$. Similarly, $F^{-1}(1)$ is defined as the right endpoint of the support of $F$, i.e. $F^{-1}(1)=\inf \{x \in \mathbb{R} \mid F(x)=1\}$, with the convention $\inf \emptyset=+\infty$.

Recall that given the random variables $X$ and $Y$ with distribution functions $F_{X}$ and $F_{Y}$ and inverses $F_{X}^{-1}$ and $F_{Y}^{-1}$, respectively, $X$ is said to be smaller than $Y$ in the dispersive 
order (denoted as $X \preceq$ disp $Y$ ) if

$$
\begin{gathered}
F_{X}^{-1}(\beta)-F_{X}^{-1}(\alpha) \leq F_{Y}^{-1}(\beta)-F_{Y}^{-1}(\alpha) \text { whenever } 0<\alpha \leq \beta<1 \\
\Leftrightarrow \alpha \mapsto F_{Y}^{-1}(\alpha)-F_{X}^{-1}(\alpha) \text { non-decreasing on }[0,1] .
\end{gathered}
$$

It is clear that the order $\preceq_{\text {disp }}$ indeed corresponds to a comparison of $X$ and $Y$ by variability because it requires the difference between any two quantiles of $X$ to be smaller than the corresponding difference in quantiles of $Y$. It is easy to prove that $X \preceq$ disp $Y$ implies $\operatorname{Var}[X] \leq \mathbb{V} \operatorname{ar}[Y]$. In addition to the definition (2.1), the following characterization is useful:

$$
X \preceq_{\text {disp }} Y \Leftrightarrow x \mapsto F_{Y}^{-1}\left(F_{X}(x)\right)-x \text { non-decreasing. }
$$

See formula (3.B.10) in Shaked and Shanthikumar (2007).

\section{Unit uniform marginals}

If the support of the conditionals is finite with common left and right endpoints then it is easy to see that the dispersive order cannot hold. This can be deduced from (2.1): considering two different distribution functions $F_{X}$ and $F_{Y}, F_{Y}^{-1}-F_{X}^{-1}$ cannot be monotone on $[0,1]$ if $F_{X}^{-1}(0)=F_{Y}^{-1}(0)>-\infty$ and $F_{X}^{-1}(1)=F_{Y}^{-1}(1)<+\infty$. Therefore, in case the support is bounded, we need different endpoints for a possible comparison in terms of the dispersive order. This is only possible if the generator is non-strict, as shown next.

Let $\left(U_{1}, U_{2}\right)$ be a couple of random variables with joint distribution function $C_{\phi}$ given in (1.3). If the generator $\phi$ has a first derivative that can be inverted then the distribution function of $\left[U_{1} \mid U_{2}=u_{2}\right]$ is given by

$$
\operatorname{Pr}\left[U_{1} \leq u_{1} \mid U_{2}=u_{2}\right]=\left\{\begin{array}{l}
\frac{\phi^{\prime}\left(u_{2}\right)}{\phi^{\prime} \circ \phi^{-1}\left(\phi\left(u_{1}\right)+\phi\left(u_{2}\right)\right)} \text { if } u_{1} \geq \phi^{-1}\left(\phi(0)-\phi\left(u_{2}\right)\right), \\
0 \text { if } u_{1}<\phi^{-1}\left(\phi(0)-\phi\left(u_{2}\right)\right)
\end{array}\right.
$$

and the corresponding quantile function is given by

$$
\psi_{u_{2}}(\alpha)=\left\{\begin{array}{l}
\phi^{-1}\left(\phi\left(\left(\phi^{\prime}\right)^{-1}\left(\frac{\phi^{\prime}\left(u_{2}\right)}{\alpha}\right)\right)-\phi\left(u_{2}\right)\right) \text { if } \alpha \geq \frac{\phi^{\prime}\left(u_{2}\right)}{\phi^{\prime}\left(0^{+}\right)} \\
\phi^{-1}\left(\phi(0)-\phi\left(u_{2}\right)\right) \text { if } \alpha<\frac{\phi^{\prime}\left(u_{2}\right)}{\phi^{\prime}\left(0^{+}\right)} .
\end{array}\right.
$$

Note that for strict generator the quantile function simplifies to

$$
\psi_{u_{2}}(\alpha)=\phi^{-1}\left(\phi\left(\left(\phi^{\prime}\right)^{-1}\left(\frac{\phi^{\prime}\left(u_{2}\right)}{\alpha}\right)\right)-\phi\left(u_{2}\right)\right) .
$$

Now, for $\alpha=1$ we get $\phi^{-1}(0)=1$ whatever $\phi$ (be it strict or not) and the conditioning value $u_{2}$. On the contrary, letting $\alpha$ tend to 0 gives 0 if $\phi$ is strict, whatever $u_{2}$, but the limit may depend on $\phi$ and on $u_{2}$ if $\phi$ is non-strict. More precisely, when $\phi$ is non strict the support is $\left[\phi^{-1}\left(\phi(0)-\phi\left(u_{2}\right)\right), 1\right]$. Therefore, for a strict generator $\phi$, the support for both $\left[U_{1} \mid U_{2}=u_{2}\right]$ and $\left[U_{1} \mid U_{2}=u_{2}^{\prime}\right]$ is the interval $[0,1]$ and no dispersive order relation can hold whereas if $\phi$ is non strict then a dispersive comparison may be possible. This is why we restrict our analysis to non-strict generators in this section devoted to unit uniform marginals. 
Remark 3.1. Note that, as stated above, the expression derived for $\psi_{u_{2}}$ requires differentiability of the generator $\phi$ and invertibility of its derivative. These conditions are not always fulfilled. For instance, the lower Frechet-Hoeffding bound generated by $\phi(t)=1-t$ does not satisfy these requirements, hence the above formula does not apply. In this particular case, the conditional distribution of $U_{1}$ given $U_{2}=u_{2}$ is degenerated at $1-u_{2}$.

We also consider conditionals of the form $\left[U_{1} \mid U_{2} \leq u_{2}\right]$ in this paper. The distribution function of $\left[U_{1} \mid U_{2} \leq u_{2}\right]$ is

$$
\operatorname{Pr}\left[U_{1} \leq u_{1} \mid U_{2} \leq u_{2}\right]=\left\{\begin{array}{l}
\frac{\phi^{-1}\left(\phi\left(u_{1}\right)+\phi\left(u_{2}\right)\right)}{u_{2}} \text { if } u_{1} \geq \phi^{-1}\left(\phi(0)-\phi\left(u_{2}\right)\right) \\
0 \text { if } u_{1} \leq \phi^{-1}\left(\phi(0)-\phi\left(u_{2}\right)\right)
\end{array}\right.
$$

The corresponding quantile function is given by

$$
\psi_{u_{2}}^{*}(\alpha)=\phi^{-1}\left(\phi\left(u_{2} \alpha\right)-\phi\left(u_{2}\right)\right)
$$

Also here, we see that for $\alpha=1$ we get $\phi^{-1}(0)=1$ whereas the limit for $\alpha$ tending to 0 is 0 for a strict generator but may depend on $\phi$ and on $u_{2}$ if the generator is non-strict. Hence, no dispersive order relation is possible between $\left[U_{1} \mid U_{2} \leq u_{2}\right]$ and $\left[U_{1} \mid U_{2} \leq u_{2}^{\prime}\right]$ if $\phi$ is strict. Here also, we need to restrict our analysis to non-strict generators.

The next result investigates the effect of increasing one component of the archimedean vector.

Proposition 3.2. Let $\left(U_{1}, U_{2}\right)$ be a couple of unit uniform random variables with archimedean copula with non-strict generator $\phi$. Assume that $\phi$ possesses a first derivative that can be inverted. Then,

(i) The stochastic inequality $\left[U_{1} \mid U_{2}=u_{2}\right] \preceq_{\text {disp }}\left[U_{1} \mid U_{2}=u_{2}^{\prime}\right]$ holds for $u_{2}<u_{2}^{\prime} \in[0,1]$ if, and only if, $\alpha \mapsto \psi_{u_{2}^{\prime}} \circ \psi_{u_{2}}^{-1}(\alpha)-\alpha$ is non-decreasing. If $\phi$ admits a second derivative then this is the case if, and only if, $\frac{\partial}{\partial \alpha} \psi_{u_{2}}(\alpha) \leq \frac{\partial}{\partial \alpha} \psi_{u_{2}^{\prime}}(\alpha)$ for all $\alpha$.

(ii) The stochastic inequality $\left[U_{1} \mid U_{2} \leq u_{2}\right] \preceq_{\text {disp }}\left[U_{1} \mid U_{2} \leq u_{2}^{\prime}\right]$ holds for $u_{2}<u_{2}^{\prime} \in[0,1]$ if, and only if, $\alpha \mapsto \psi_{u_{2}^{\prime}}^{*} \circ \psi_{u_{2}}^{*-1}(\alpha)-\alpha$ is non-decreasing or, equivalently, if, and only if, $\frac{\partial}{\partial \alpha} \psi_{u_{2}}^{*}(\alpha) \leq \frac{\partial}{\partial \alpha} \psi_{u_{2}^{\prime}}^{*}(\alpha)$ for all $\alpha$.

Proof. The first part of the statements in (i)-(ii) is a direct application of (2.2). To prove the second part of the statement in (i), note that

$$
\begin{aligned}
& \psi_{u_{2}^{\prime}} \circ \psi_{u_{2}}^{-1}(\alpha)-\alpha \text { is non-decreasing in } \alpha \\
\Leftrightarrow & \frac{\frac{\partial}{\partial \alpha} \psi_{u_{2}^{\prime}}\left(\psi_{u_{2}}^{-1}(\alpha)\right)-\frac{\partial}{\partial \alpha} \psi_{u_{2}}\left(\psi_{u_{2}}^{-1}(\alpha)\right)}{\frac{\partial}{\partial \alpha} \psi_{u_{2}}\left(\psi_{u_{2}}^{-1}(\alpha)\right)} \geq 0 \text { for all } \alpha \\
\Leftrightarrow & \frac{\partial}{\partial \alpha} \psi_{u_{2}^{\prime}}\left(\psi_{u_{2}}^{-1}(\alpha)\right)-\frac{\partial}{\partial \alpha} \psi_{u_{2}}\left(\psi_{u_{2}}^{-1}(\alpha)\right) \geq 0 \text { for all } \alpha \\
\Leftrightarrow & \frac{\partial}{\partial \alpha} \psi_{u_{2}}(\alpha) \leq \frac{\partial}{\partial \alpha} \psi_{u_{2}^{\prime}}(\alpha) \text { for all } \alpha
\end{aligned}
$$

which ends the proof of (i). The reasoning leading to (ii) is similar. 
Let us now examine an example where Proposition 3.2 applies.

Example 3.3 (Family 7 in Table 4.1 of Nelsen (2006)). Consider the generator $\phi_{\theta}(t)=$ $-\ln (\theta t+(1-\theta))$ indexed by $\theta \in(0,1]$. The corresponding copula function is

$$
C_{\dot{\phi}_{\theta}}\left(u_{1}, u_{2}\right)=\max \left\{\theta u_{1} u_{2}+(1-\theta)\left(u_{1}+u_{2}-1\right), 0\right\} .
$$

Consider $\left(U_{1}, U_{2}\right)$ with joint distribution function $C_{\phi_{\theta}}$. For $\theta=0$, we get the lower FrechetHoeffding bound copula $C\left(u_{1}, u_{2}\right)=\max \left\{u_{1}+u_{2}-1,0\right\}$ which gives the minimum in the dispersive sense since $\left[U_{1} \mid U_{2}=u_{2}\right]$ is constantly equal to $1-u_{2}$ in that case. For $\theta=1$, we get the independent case.

Direct calculations yield

$$
\begin{aligned}
\phi^{\prime}\left(u_{2}\right) & =\frac{-\theta}{\theta u_{2}+1-\theta} \\
\left(\phi^{\prime}\right)^{-1}(x) & =-\frac{1}{x}-\frac{1-\theta}{\theta} \\
\left(\phi^{\prime}\right)^{-1}\left(\frac{\phi^{\prime}\left(u_{2}\right)}{\alpha}\right) & =\frac{\alpha\left(\theta u_{2}+1-\theta\right)+\theta-1}{\theta} \\
\phi\left(\left(\phi^{\prime}\right)^{-1}\left(\frac{\phi^{\prime}\left(u_{2}\right)}{\alpha}\right)\right)-\phi\left(u_{2}\right) & =-\ln (\alpha) .
\end{aligned}
$$

Now, since

$$
\phi^{-1}(x)=\frac{e^{-x}+\theta-1}{\theta}
$$

we have

$$
\phi^{-1}\left(\phi\left(\left(\phi^{\prime}\right)^{-1}\left(\frac{\phi^{\prime}\left(u_{2}\right)}{\alpha}\right)\right)-\phi\left(u_{2}\right)\right)=\frac{\alpha}{\theta}-\frac{1-\theta}{\theta}
$$

Also,

$$
\frac{\phi^{\prime}\left(u_{2}\right)}{\phi^{\prime}\left(0^{+}\right)}=\frac{1-\theta}{\theta u_{2}+1-\theta} \text { and } \phi^{-1}\left(\phi(0)-\phi\left(u_{2}\right)\right)=\frac{\left(1-u_{2}\right)(1-\theta)}{\theta u_{2}+1-\theta} \text {. }
$$

Finally,

$$
\psi_{u_{2}}(\alpha)=\left\{\begin{array}{l}
\frac{\alpha-1+\theta}{\theta} \text { if } \alpha \geq \frac{1-\theta}{\theta u_{2}+1-\theta}, \\
\frac{\left(1-u_{2}\right)(1-\theta)}{\theta u_{2}+1-\theta} \text { if } \alpha<\frac{1-\theta}{\theta u_{2}+1-\theta}
\end{array}\right.
$$

so that for $t^{\prime}<t$,

$$
\psi_{t^{\prime}}(\alpha)-\psi_{t}(\alpha)=\left\{\begin{array}{l}
0 \text { if } \alpha \geq \frac{1-\theta}{\theta t^{\prime}+1-\theta}, \\
\frac{(1-\theta)\left(1-t^{\prime}\right)}{\theta t^{\prime}+1-\theta}-\frac{\alpha-1+\theta}{\theta} \text { if } \frac{1-\theta}{\theta t+1-\theta} \leq \alpha \leq \frac{1-\theta}{\theta t^{\prime}+1-\theta}, \\
\frac{(1-\theta)\left(1-t^{\prime}\right)}{\theta t^{\prime}+1-\theta}-\frac{(1-\theta)(1-t)}{\theta t+1-\theta} \text { if } \alpha \leq \frac{1-\theta}{\theta t+1-\theta} .
\end{array}\right.
$$

Since $\psi_{t^{\prime}}-\psi_{t}$ is non-increasing in $\alpha$, we finally get by $(2.1)$

$$
u_{2}<u_{2}^{\prime} \quad \Rightarrow \quad\left[U_{1} \mid U_{2}=u_{2}\right] \preceq_{\operatorname{disp}}\left[U_{1} \mid U_{2}=u_{2}^{\prime}\right] .
$$


For this copula, we thus see that increasing the second component increases the conditional distribution in the dispersive order. Hence, $U_{1}$ becomes more variable when $U_{2}$ ages so that the copula $C_{\phi_{\theta}}$ induces some positive cross-aging variability effect.

Now, as

$$
\frac{\partial}{\partial \alpha} \psi_{t}^{*}(\alpha)=\frac{t}{\theta t+1-\theta}
$$

increases in $t$, we also have

$$
u_{2}<u_{2}^{\prime} \quad \Rightarrow \quad\left[U_{1} \mid U_{2} \leq u_{2}\right] \preceq_{\text {disp }}\left[U_{1} \mid U_{2} \leq u_{2}^{\prime}\right] .
$$

The knowledge that $U_{2}$ falls below a smaller threshold $u_{2}$ compared to $u_{2}^{\prime}$ thus decreases the variability of $U_{1}$. In other words, allowing $U_{2}$ to assume larger values (less than $u_{2}^{\prime}$ instead of less than $u_{2}$ ) makes $U_{1}$ more variable in the $\preceq_{\text {disp }}$-sense.

\section{Arbitrary marginals}

\subsection{Concave marginal distribution function}

Now that we have derived in Proposition 3.2 an effective condition for the conditionals to be ordered in the dispersive order for the unit uniform case, it is natural to wonder whether this condition also applies to random couples with arbitrary marginals connected through an archimedean copula. However, the results obtained in Section 3 do not allow to treat this more general situation. The reason is that the implication $X \preceq_{\text {disp }} Y \Rightarrow g(X) \preceq_{\text {disp }} g(Y)$ is not necessarily true for increasing transformations $g$ unless additional assumptions about the shape of the function $g$ and the respective distributions of $X$ and $Y$ are fulfilled.

There is nevertheless one particular case where the results derived in Section 3 extend to other marginals than unit uniform ones, as discussed next.

Proposition 4.1. Let $\left(X_{1}, X_{2}\right)$ be a random vector with distribution function (1.2) such that $F_{1}$ and $F_{2}$ are continuous with supports contained in $\mathbb{R}^{+}$. Assume that $\phi$ has a first derivative that can be inverted. Then,

(i) If $F_{1}$ is concave on $\mathbb{R}^{+}$then $\left[X_{1} \mid X_{2}=x_{2}\right]$ increases in $x_{2}$ in the $\preceq$ disp-sense if the non-strict generator $\phi$ fulfills the condition of Proposition 3.2(i).

(ii) If $F_{1}$ is concave on $\mathbb{R}^{+}$then $\left[X_{1} \mid X_{2} \leq x_{2}\right]$ increases in $x_{2}$ in the $\preceq$ disp-sense if the non-strict generator $\phi$ fulfills the condition of Proposition 3.2(ii).

Proof. Let us establish (i). If we define $U_{i}=F_{i}\left(X_{i}\right), i=1,2$, then $\left(U_{1}, U_{2}\right)$ fulfills the conditions of Proposition 3.2(i). Consider $u_{2}<u_{2}^{\prime} \in[0,1]$. Since the common right endpoint of the supports of $\left[U_{1} \mid U_{2}=u_{2}\right]$ and of $\left[U_{1} \mid U_{2}=u_{2}^{\prime}\right]$ is 1 , we have $\left[U_{1} \mid U_{2}=u_{2}^{\prime}\right] \preceq_{\text {st }}\left[U_{1} \mid U_{2}=\right.$ $\left.u_{2}\right]$. Also, we have from Proposition 3.2(i) that $\left[U_{1} \mid U_{2}=u_{2}\right] \preceq_{\text {disp }}\left[U_{1} \mid U_{2}=u_{2}^{\prime}\right]$. From Theorem 3.B.10 in Shaked and Shanthikumar (2007), we see that provided and $F_{1}^{-1}$ is convex (or, equivalently, $F_{1}$ is concave, that is, the corresponding probability density function is 
decreasing), we have

$$
\begin{aligned}
& {\left[U_{1} \mid U_{2}=u_{2}\right] \preceq_{\text {disp }}\left[U_{1} \mid U_{2}=u_{2}^{\prime}\right] \text { for all } u_{2}<u_{2}^{\prime} \in[0,1] } \\
\Rightarrow & {\left[F_{1}^{-1}\left(U_{1}\right) \mid U_{2}=u_{2}\right] \preceq_{\text {disp }}\left[F_{1}^{-1}\left(U_{1}\right) \mid U_{2}=u_{2}^{\prime}\right] \text { for all } u_{2}<u_{2}^{\prime} \in[0,1] } \\
\Leftrightarrow & {\left[X_{1} \mid X_{2}=x_{2}\right] \preceq_{\text {disp }}\left[X_{1} \mid X_{2}=x_{2}^{\prime}\right] \text { for all } x_{2}<x_{2}^{\prime} \text { in the support of } X_{2} . }
\end{aligned}
$$

The same reasoning shows that (ii) also holds true.

Concave distribution functions are unimodal about 0 (i.e. they possess decreasing densities). For such distributions, the assumptions of Proposition 3.2 are thus enough to ensure

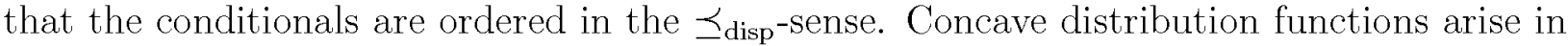
a number of ways in applied probability. In particular, all the DFR (for decreasing failure rate) distributions have concave distribution functions. Moreover, this class is closed under change of scale, power transformation, left truncation, limits, mixtures and the formation of arbitrary series systems.

\subsection{General case}

Let us now consider arbitrary marginals. Note that switching from unit uniform to arbitrary marginals allows us to consider a strict generator $\phi$ as long as the supports of the conditional distributions do not coincide with some bounded interval. For instance, considering a strict generator $\phi$ with marginals $F_{1}$ and $F_{2}$ with common support $(0,+\infty)$ makes a $\preceq_{\text {disp }}$ comparison possible. For these reasons, we do not repeat the conditions on the generator, keeping in mind that we exclude the case with identical bounded supports in the next result.

Proposition 4.2. Let $\boldsymbol{X}=\left(X_{1}, X_{2}\right)$ be a random vector with distribution function (1.2) such that $F_{1}$ and $F_{2}$ are continuous and strictly increasing. Assume that $\phi$ has a first derivative that can be inverted. Then,

(i) the stochastic inequality $\left[X_{1} \mid X_{2}=x_{2}\right] \preceq_{\text {disp }}\left[X_{1} \mid X_{2}=x_{2}^{\prime}\right]$ holds for $x_{2}<x_{2}^{\prime}$ if, and only if,

$$
\alpha \mapsto F_{1}^{-1}\left(\psi_{F_{2}\left(x_{2}^{\prime}\right)}(\alpha)\right)-F_{1}^{-1}\left(\psi_{F_{2}\left(x_{2}\right)}(\alpha)\right) \text { is non-decreasing on }[0,1] \text {. }
$$

(ii) the stochastic inequality $\left[X_{1} \mid X_{2} \leq x_{2}\right] \preceq_{\text {disp }}\left[X_{1} \mid X_{2} \leq x_{2}^{\prime}\right]$ holds for $x_{2}<x_{2}^{\prime}$ if, and only if,

$$
\alpha \mapsto F_{1}^{-1}\left(\psi_{F_{2}\left(x_{2}^{\prime}\right)}^{*}(\alpha)\right)-F_{1}^{-1}\left(\psi_{F_{2}\left(x_{2}\right)}^{*}(\alpha)\right) \text { is non-decreasing on }[0,1] \text {. }
$$

Proof. The result is a consequence of (2.1). Define $U_{i}=F_{i}\left(X_{i}\right), i=1,2$. Considering (i), the conditional distribution of $X_{1}$ is given by

$$
\begin{aligned}
\operatorname{Pr}\left[X_{1} \leq x_{1} \mid X_{2}=x_{2}\right] & =\operatorname{Pr}\left[F_{1}^{-1}\left(U_{1}\right) \leq x_{1} \mid F_{2}^{-1}\left(U_{2}\right)=x_{2}\right] \\
& =\operatorname{Pr}\left[U_{1} \leq F_{1}\left(x_{1}\right) \mid U_{2}=F_{2}\left(x_{2}\right)\right] \\
& =\psi_{F_{2}\left(x_{2}\right)}^{-1}\left(F_{1}\left(x_{1}\right)\right)
\end{aligned}
$$

so that the corresponding quantile function is $F_{1}^{-1} \circ \psi_{F_{2}\left(x_{2}\right)}$. The proof for (ii) is similar. 
Note that only $F_{1}$ matters in Proposition 4.2 , not $F_{2}$. This comes from the fact that the condition $X_{2}=x_{2}$ or $X_{2} \leq x_{2}$ can equivalently be expressed in terms of $U_{2}=F_{2}\left(X_{2}\right)$, coming back to the unit uniform distribution whatever $F_{2}$.

If $\phi$ possesses a second derivative then another way to state the results in Proposition 4.2 consists in imposing that the first derivative of (4.1)-(4.2) is non-negative. For instance, this gives for (4.1)

$$
x_{2} \mapsto \frac{\frac{\partial}{\partial \alpha} \psi_{F_{2}\left(x_{2}\right)}}{f_{1}\left(F_{1}^{-1}\left(\psi_{F_{2}\left(x_{2}\right)}(\alpha)\right)\right)} \text { non-decreasing, }
$$

where $f_{1}$ denotes the probability density function corresponding to $F_{1}$.

\subsection{Examples}

Let us now consider a couple of examples involving standard families of parametric archimedean copulas.

Example 4.3. Consider Frank's copula given by

$$
C_{\phi_{\theta}}\left(u_{1}, u_{2}\right)=-\frac{1}{\theta} \ln \left(1+\frac{\left(\exp \left(-\theta u_{1}\right)-1\right)\left(\exp \left(-\theta u_{2}\right)-1\right)}{\exp (-\theta)-1}\right), \theta \neq 0 .
$$

This is an archimedean copula with generator $\phi_{\theta}(t)=\ln \left(e^{-\theta}-1\right)-\ln \left(e^{-t \theta}-1\right)$. Then, we obtain

$$
\psi_{F_{2}\left(x_{2}\right)}(\alpha)=\frac{1}{\theta} \ln \left(\frac{e^{-\theta F_{2}\left(x_{2}\right)}+\alpha\left(1-e^{-\theta F_{2}\left(x_{2}\right)}\right)}{e^{-\theta F_{2}\left(x_{2}\right)}+\alpha\left(e^{-\theta}-e^{-\theta F_{2}\left(x_{2}\right)}\right)}\right)
$$

and

$$
\psi_{F_{2}\left(x_{2}\right)}^{*}(\alpha)=\frac{1}{\theta} \ln \left(\frac{e^{-\theta F_{2}\left(x_{2}\right)}-1}{e^{-\theta F_{2}\left(x_{2}\right)}-1+\left(e^{-\theta F_{2}\left(x_{2}\right) \alpha}-1\right)\left(e^{-\theta}-1\right)}\right) .
$$

For instance, with unit Exponential marginal $F_{1}$, that is, $F_{1}(x)=1-\exp (-x)$, we get

$$
F_{1}^{-1}\left(\psi_{F_{2}\left(x_{2}^{\prime}\right)}(\alpha)\right)-F_{1}^{-1}\left(\psi_{F_{2}\left(x_{2}\right)}(\alpha)\right)=-\ln \left(\frac{1-\psi_{F_{2}\left(x_{2}^{\prime}\right)}(\alpha)}{1-\psi_{F_{2}\left(x_{2}\right)}(\alpha)}\right)
$$

and

$$
F_{1}^{-1}\left(\psi_{F_{2}\left(x_{2}^{\prime}\right)}^{*}(\alpha)\right)-F_{1}^{-1}\left(\psi_{F_{2}\left(x_{2}\right)}^{*}(\alpha)\right)=-\ln \left(\frac{1-\psi_{F_{2}\left(x_{2}^{\prime}\right)}^{*}(\alpha)}{1-\psi_{F_{2}\left(x_{2}\right)}^{*}(\alpha)}\right) .
$$

For $\theta \geq 0$, Frank's copulas express positive dependence, i.e. large values of one component tend to be associated with large values of the other one. Considering $F_{2}\left(x_{2}\right)=0.25$ and $F_{2}\left(x_{2}^{\prime}\right)=0.75$, Figure 4.1 (top left panel) shows that the difference (4.5) is increasing for values of $\theta$ corresponding to Kendall's $\tau$ equal to $0.1,0.4,0.7$, and 0.9 , respectively. This means that increasing the value of $X_{2}$ makes $X_{1}$ more variable in the $\preceq_{\text {disp }}$-sense. On the contrary, for $\theta \leq 0$, the dependence is negative, that is, large values of one component tend to be associated with small values of the other one. For such $\theta$ s, we see from Figure 4.1 (top 
right panel) that the difference (4.5) is now decreasing. Increasing $X_{2}$ now makes $X_{1}$ less variable in the $\preceq_{\text {disp }}$-sense. Moving from the center of the distribution to the tails does not modify the conclusion, as it can be seen from Figure 4.2 (top panels) where we consider $x_{2}$ and $x_{2}^{\prime}$ such that $F_{2}\left(x_{2}\right)=0.99$ and $F_{2}\left(x_{2}^{\prime}\right)=0.995$. Figure 4.3 shows the difference (4.5) as a function of $\alpha$ and $\theta$. The different behavior according to the sign of $\theta$ is clearly visible there.

In addition to the unit Exponential case, we also consider in Figures 4.1-4.2 the case of Pareto marginal $F_{1}$, that is, $F_{1}(x)=1-x^{-a}$ for $x>1$ and some $a>0$, standard Normal marginal $F_{1}$, and Gamma marginal $F_{1}$. We can see there that the results obtain in the unit Exponential case are also valid in the Pareto case. However, no dispersive order relation holds in the Normal case whereas in the Gamma case, the dispersive order relation is valid only for sufficiently high value of Kendall's correlation coefficient. This illustrates the effect of marginal distributions on cross-aging.

Example 4.4. Consider Clayton's copula defined by

$$
C_{\phi_{\theta}}\left(u_{1}, u_{2}\right)=\left(u_{1}^{-\theta}+u_{2}^{-\theta}-1\right)^{-1 / \theta}, \theta>0 .
$$

This copula belongs to the archimedean class, generated by $\phi_{\theta}(t)=\frac{t^{-\theta}-1}{\theta}, \theta>0$, which is strict. In this case, we find $\phi_{\theta}^{-1}(t)=(\theta t+1)^{-1 / \theta}$ and

$$
\psi_{F_{2}\left(x_{2}\right)}(\alpha)=\left(1+\left(F_{2}\left(x_{2}\right)\right)^{-\theta}\left(\alpha^{-\theta /(\theta+1)}-1\right)\right)^{-1 / \theta}
$$

and

$$
\psi_{F_{2}\left(x_{2}\right)}^{*}(\alpha)=\left(\left(F_{2}\left(x_{2}\right) \alpha\right)^{-\theta}-F_{2}\left(x_{2}\right)^{-\theta}+1\right)^{-1 / \theta} .
$$

For instance, with Pareto marginal $F_{1}$, we get

$$
F_{1}^{-1}\left(\psi_{F_{2}\left(x_{2}^{\prime}\right)}(\alpha)\right)-F_{1}^{-1}\left(\psi_{F_{2}\left(x_{2}\right)}(\alpha)\right)=\left(1-\psi_{F_{2}\left(x_{2}^{\prime}\right)}(\alpha)\right)^{-1 / a}-\left(1-\psi_{F_{2}\left(x_{2}\right)}(\alpha)\right)^{-1 / a}
$$

and

$$
F_{1}^{-1}\left(\psi_{F_{2}\left(x_{2}^{\prime}\right)}^{*}(\alpha)\right)-F_{1}^{-1}\left(\psi_{F_{2}\left(x_{2}\right)}^{*}(\alpha)\right)=\left(1-\psi_{F_{2}\left(x_{2}^{\prime}\right)}^{*}(\alpha)\right)^{-1 / a}-\left(1-\psi_{F_{2}\left(x_{2}\right)}^{*}(\alpha)\right)^{-1 / a} .
$$

Note that the dependence expressed by Clayton copula (4.7) is always positive (an extension of (4.7) to negative $\theta \mathrm{s}$ is possible but is not considered here). The limiting case $\theta=0$ corresponds to independence and increasing $\theta$ strengthens the positive relationship between the two components of the random couple. Figure 4.4 is the counterpart of Figure 4.1 and Figure 4.5 is the counterpart of Figure 4.3 for Clayton copula. The conclusions drawn for Frank copulas in the case $\theta>0$ still apply to Clayton copulas. 

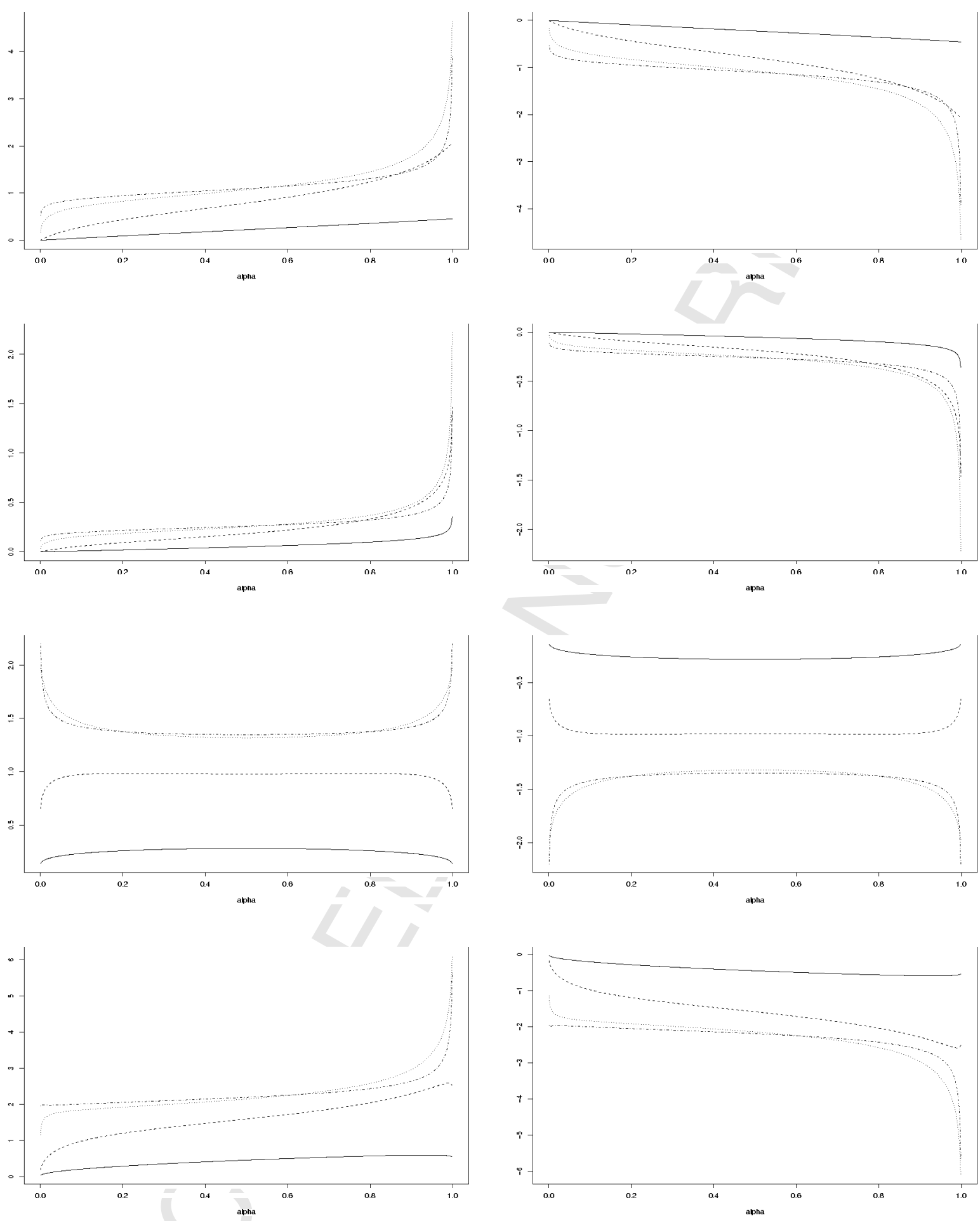

Figure 4.1: Graph of (4.1) for Frank copula with $x_{2}$ and $x_{2}^{\prime}$ such that $F_{2}\left(x_{2}\right)=0.25$ and $F_{2}\left(x_{2}^{\prime}\right)=0.75$ and values of $\theta$ corresponding to Kendall's $\tau$ equal to 0.1 (solid), 0.4 (dashed), 0.7 (dotted), and 0.9 (dotdash) in the left panels and to -0.1 (solid), -0.4 (dashed), -0.7 (dotted), and -0.9 (dotdash) in the right panels. From top to bottom: unit Exponential marginal $F_{1}$, Pareto marginal $F_{1}$ (with $a=5$ ), standard Normal marginal $F_{1}$, and Gamma marginal $F_{1}$ (with shape parameter 3 and scale parameter 1 , that is, with mean and variance equal to 3$)$. 

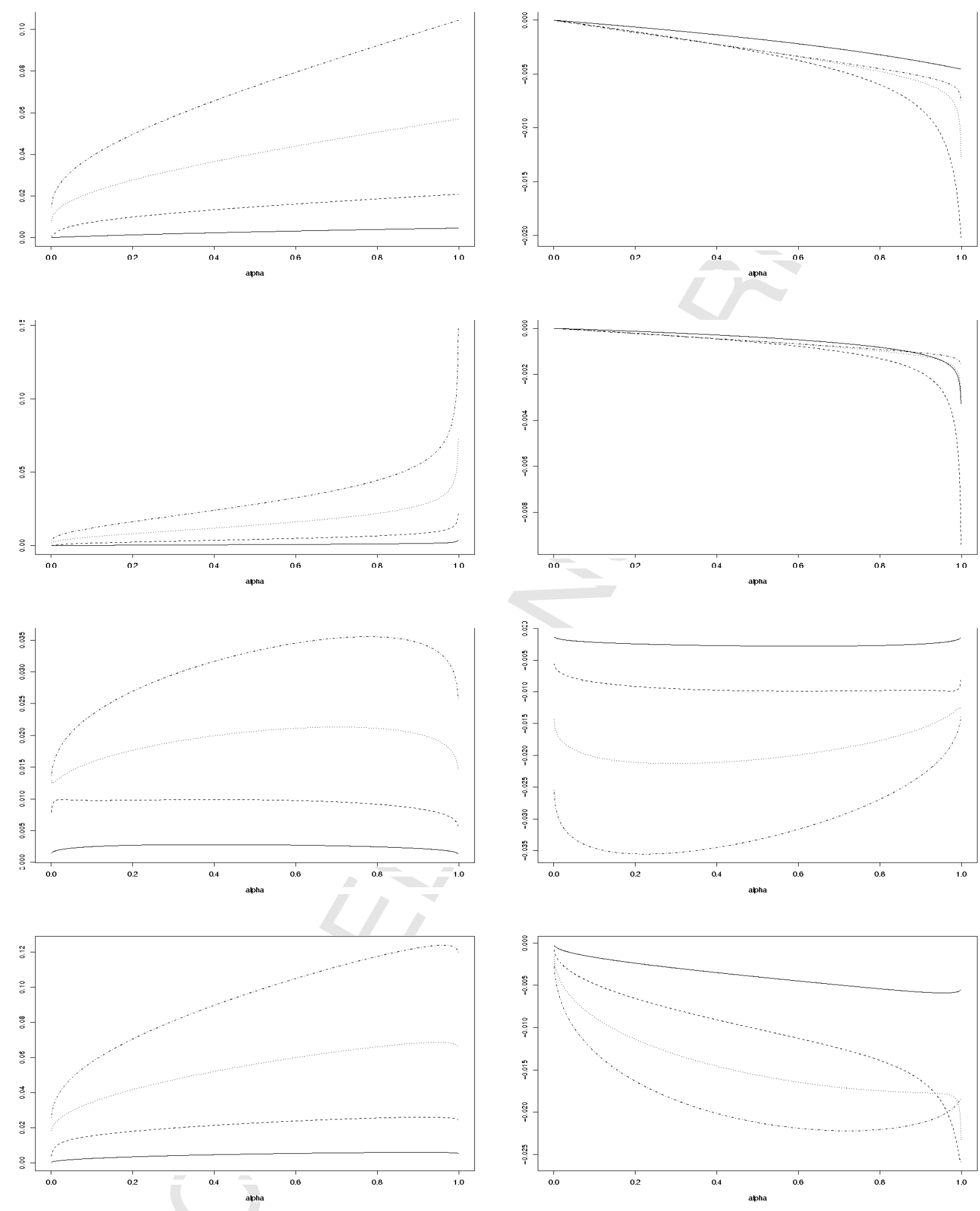

Figure 4.2: Graph of (4.1) for Frank copula with $x_{2}$ and $x_{2}^{\prime}$ such that $F_{2}\left(x_{2}\right)=0.99$ and $F_{2}\left(x_{2}^{\prime}\right)=0.995$ and values of $\theta$ corresponding to Kendall's $\tau$ equal to 0.1 (solid), 0.4 (dashed), 0.7 (dotted), and 0.9 (dotdash) in the left panels and to -0.1 (solid), -0.4 (dashed), -0.7 (dotted), and -0.9 (dotdash) in the right panels. From top to bottom: unit Exponential marginal $F_{1}$, Pareto marginal $F_{1}$ (with $a=5$ ), standard Normal marginal $F_{1}$, and Gamma marginal $F_{1}$ (with shape parameter 3 and scale parameter 1 , that is, with mean and variance equal to 3$)$. 

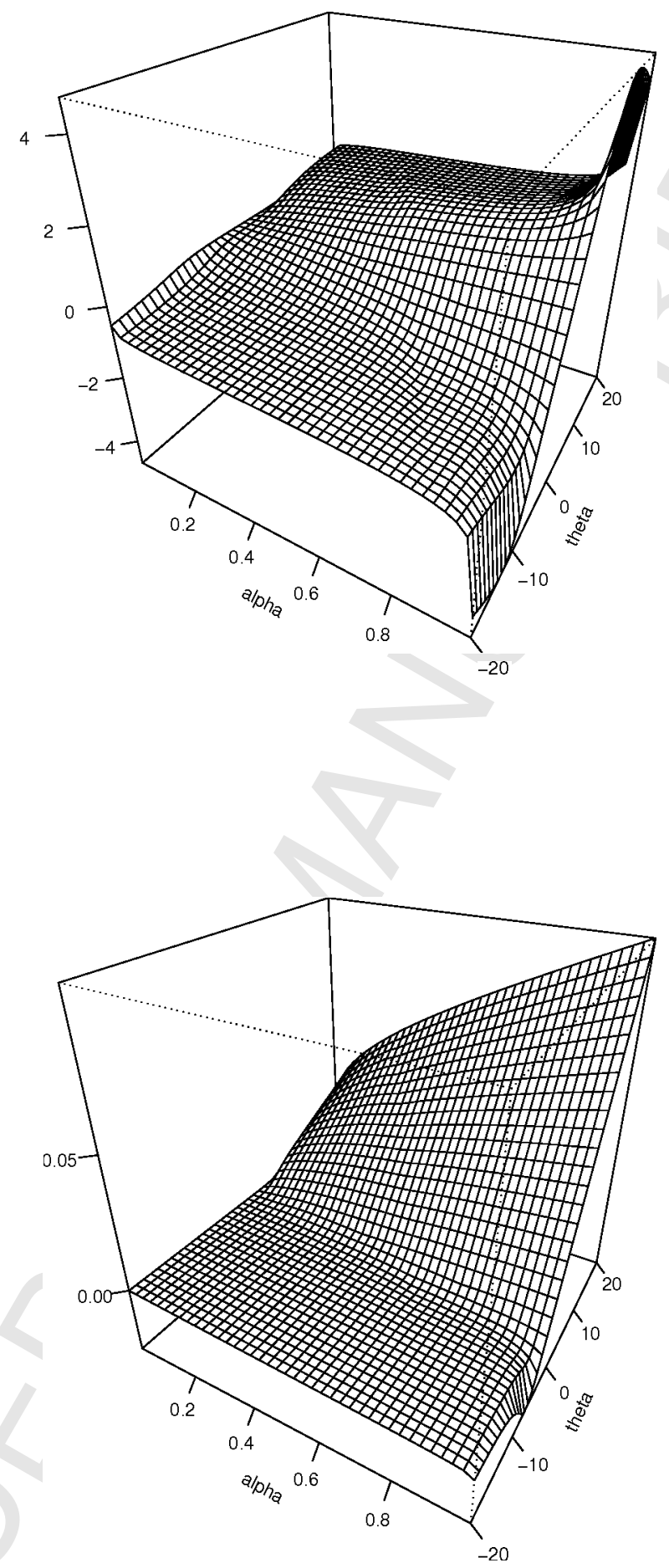

Figure 4.3: Graph of (4.5) as a function of $\alpha$ and $\theta$ for Frank copula with $x_{2}$ and $x_{2}^{\prime}$ such that $F_{2}\left(x_{2}\right)=0.25$ and $F_{2}\left(x_{2}^{\prime}\right)=0.25$ (top panel) and with $x_{2}$ and $x_{2}^{\prime}$ such that $F_{2}\left(x_{2}\right)=0.99$ and $F_{2}\left(x_{2}^{\prime}\right)=0.995$ (bottom panel) with unit Exponential marginal $F_{1}$. 

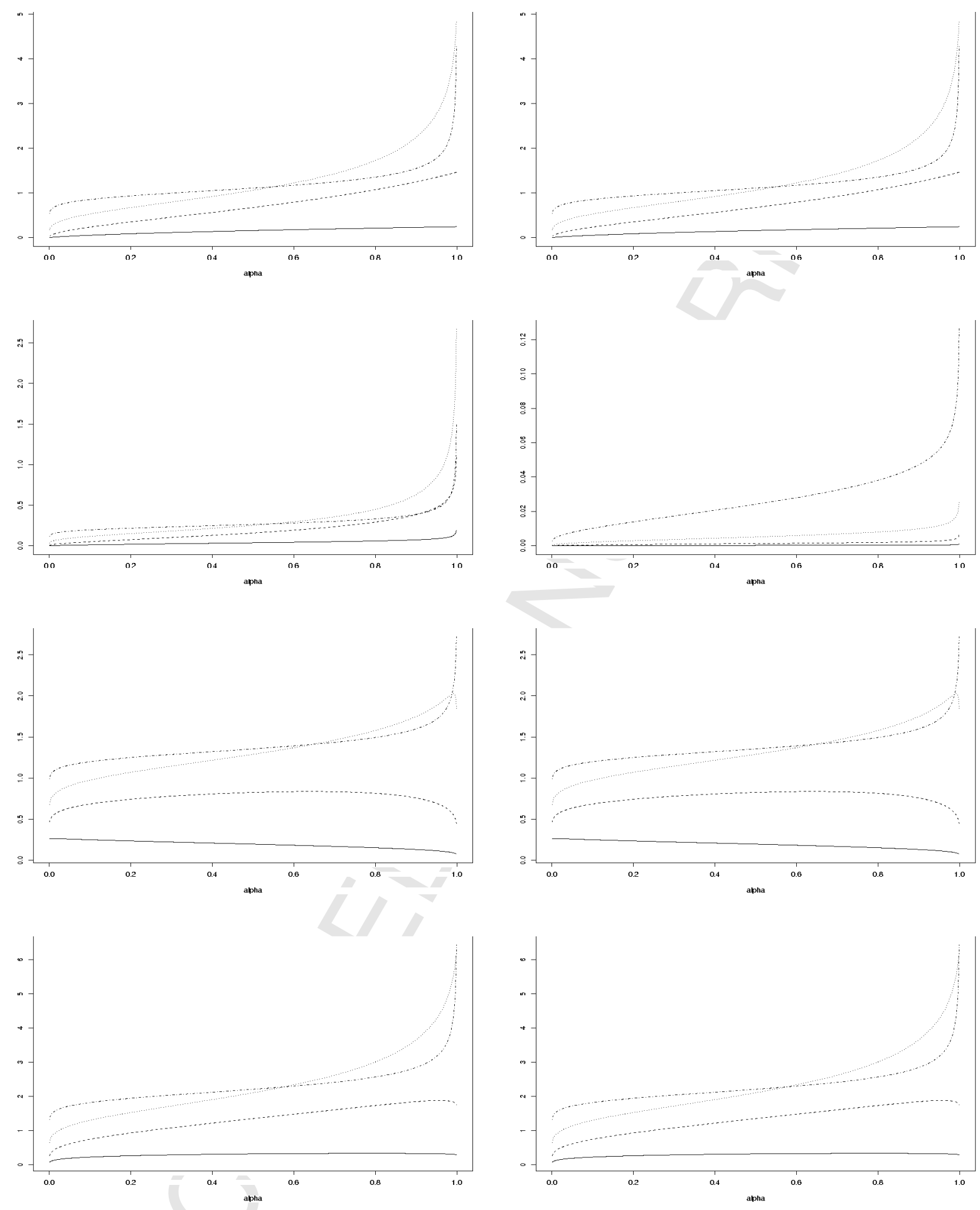

Figure 4.4: Graph of (4.1) for $F_{2}\left(x_{2}\right)=0.25$ and $F_{2}\left(x_{2}^{\prime}\right)=0.75$ (left panel) and $F_{2}\left(x_{2}\right)=$ 0.99 and $F_{2}\left(x_{2}^{\prime}\right)=0.995$ (right panel) for Clayton copula and values of $\theta$ corresponding to Kendall's $\tau$ equal to (solid), 0.4 (dashed), 0.7 (dotted), and 0.9 (dotdash). From top to bottom: unit Exponential marginal $F_{1}$, Pareto marginal $F_{1}$ (with $a=5$ ), standard Normal marginal $F_{1}$, and Gamma marginal $F_{1}$ (with shape parameter 3 and scale parameter 1 , that is, with mean and variance equal to 3 ). 

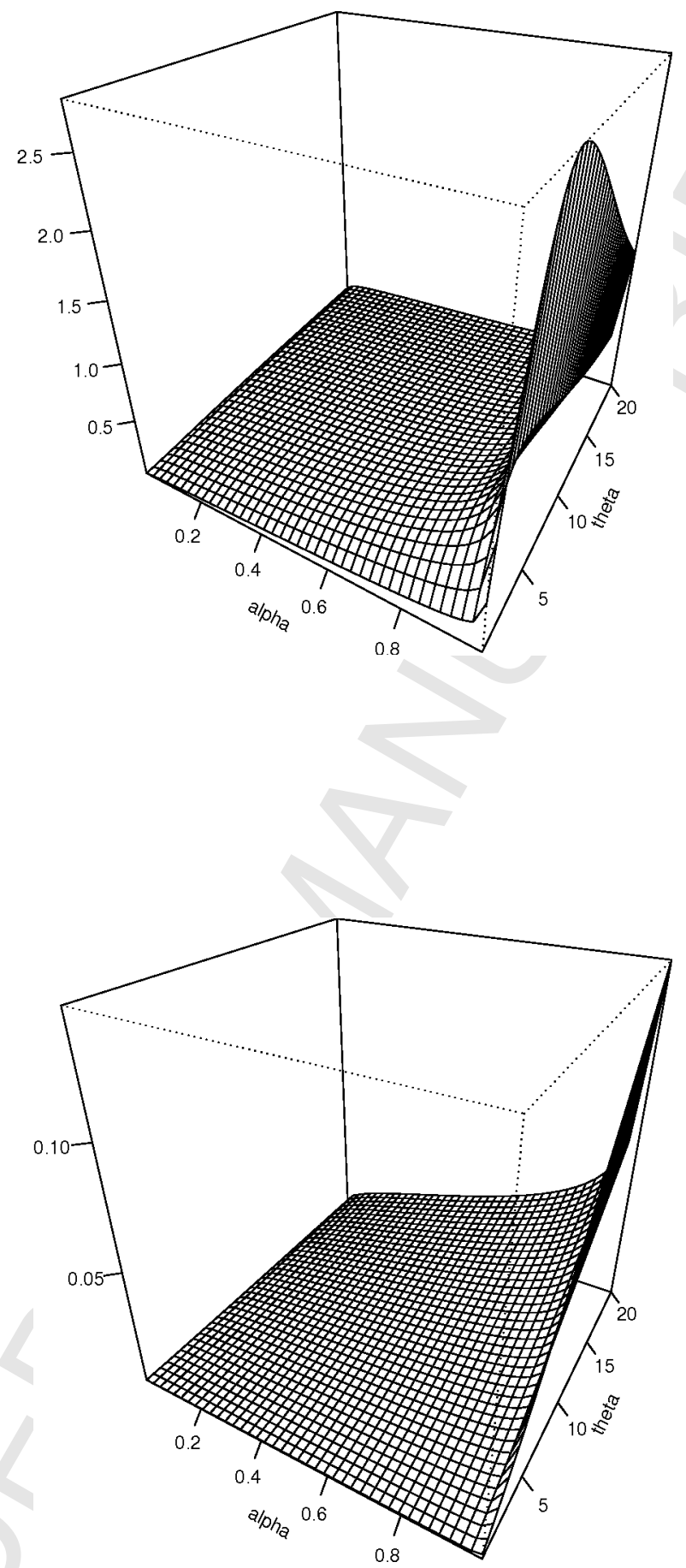

Figure 4.5: Graph of (4.10) as a function of $\alpha$ and $\theta$ for Clayton copula with $x_{2}$ and $x_{2}^{\prime}$ such that $F_{2}\left(x_{2}\right)=0.25$ and $F_{2}\left(x_{2}^{\prime}\right)=0.75$ (top panel) and with $x_{2}$ and $x_{2}^{\prime}$ such that $F_{2}\left(x_{2}\right)=0.99$ and $F_{2}\left(x_{2}^{\prime}\right)=0.995$ (bottom panel) with Pareto marginal $F_{1}(a=5)$. 


\section{Conditional comparison of random vectors with iden- tical copulas}

Consider two random couples, $\left(X_{1}, X_{2}\right)$ and $\left(Y_{1}, Y_{2}\right)$, say, sharing the same archimedean copula $C_{\phi}$. We assume that $\left(X_{1}, X_{2}\right)$ possesses the dispersive cross-aging property and we would like to compare conditional distributions $\left|X_{1}\right| X_{2}=x_{2} \mid$ and $\left|Y_{1}\right| Y_{2}=x_{2} \mid$ when the marginals are ordered. The next result provides an answer to this problem.

Proposition 5.1. Let $\left(X_{1}, X_{2}\right)$ and $\left(Y_{1}, Y_{2}\right)$ be two random couples with the same archimedean copula $C_{\phi}$ and with continuous and strictly increasing marginal distribution functions. We assume that $\phi$ possesses a first derivative that can be inverted and that $\left[X_{1} \mid X_{2}=x_{2}\right] \preceq_{\text {disp }}$ $\left[X_{1} \mid X_{2}=x_{2}^{\prime}\right]$ holds for all $x_{2} \leq x_{2}^{\prime}$. Then,

$$
X_{1} \preceq_{\text {disp }} Y_{1} \text { and } Y_{2} \preceq_{\text {st }} X_{2} \Rightarrow\left|X_{1}\right| X_{2}=x_{2}\left|\preceq_{\text {disp }}\right| Y_{1}\left|Y_{2}=x_{2}\right| \text { for all } x_{2} \text {. }
$$

Proof. Denote as $F_{i}$ the distribution function of $X_{i}, i=1,2$, and as $G_{i}$ the distribution function of $Y_{i}, i=1,2$. Clearly,

$$
\begin{aligned}
G_{1}^{-1}\left(\psi_{G_{2}\left(x_{2}\right)}(\alpha)\right)-F_{1}^{-1}\left(\psi_{F_{2}\left(x_{2}\right)}(\alpha)\right)= & \left(G_{1}^{-1}\left(\psi_{G_{2}\left(x_{2}\right)}(\alpha)\right)-F_{1}^{-1}\left(\psi_{G_{2}\left(x_{2}\right)}(\alpha)\right)\right) \\
& +\left(F_{1}^{-1}\left(\psi_{G_{2}\left(x_{2}\right)}(\alpha)\right)-F_{1}^{-1}\left(\psi_{F_{2}\left(x_{2}\right)}(\alpha)\right)\right) .(5
\end{aligned}
$$

Since $X_{1} \preceq_{\text {disp }} Y_{1}$ we know that $\alpha \mapsto G_{1}^{-1}(\alpha)-F_{1}^{-1}(\alpha)$ is non-decreasing. This, in turn, implies that $\alpha \mapsto G_{1}^{-1}\left(\psi_{G_{2}\left(x_{2}\right)}(\alpha)\right)-F_{1}^{-1}\left(\psi_{G_{2}\left(x_{2}\right)}(\alpha)\right)$ is non-decreasing, since $\alpha \mapsto \psi_{G_{2}\left(x_{2}\right)}(\alpha)$ is non-decreasing. The function inside the first bracket of $(5.1)$ is thus non-decreasing. Let us now consider the function inside the second bracket of (5.1). Putting $x_{2}^{\prime}=F_{2}^{-1}\left(G_{2}\left(x_{2}\right)\right)$, we have $x_{2} \leq x_{2}^{\prime}$ since $Y_{2} \preceq_{\text {st }} X_{2}$. Now using the fact that $\left[X_{1} \mid X_{2}=x_{2}\right] \preceq_{\text {disp }}\left[X_{1} \mid X_{2}=\right.$ $\left.x_{2}^{\prime}\right]$, we see that $F_{1}^{-1}\left(\psi_{F_{2}\left(x_{2}^{\prime}\right)}(\alpha)\right)-F_{1}^{-1}\left(\psi_{F_{2}\left(x_{2}\right)}(\alpha)\right)$ is non-decreasing and coincides with $F_{1}^{-1}\left(\psi_{G_{2}\left(x_{2}\right)}(\alpha)\right)-F_{1}^{-1}\left(\psi_{F_{2}\left(x_{2}\right)}(\alpha)\right.$. Hence, $G_{1}^{-1}\left(\psi_{G_{2}\left(x_{2}\right)}(\alpha)\right)-F_{1}^{-1}\left(\psi_{F_{2}\left(x_{2}\right)}(\alpha)\right)$ appears as the sum of two non-decreasing functions and is therefore also non-decreasing, which ends the proof.

A similar result holds for conditional distributions $\left[X_{1} \mid X_{2} \leq x_{2}\right]$ and $\left[Y_{1} \mid Y_{2} \leq x_{2}\right]$. Taking $F_{2}=G_{2}$, we see that increasing the first marginal distribution in the $\preceq_{\text {disp }}$-sense also increases the conditional distributions in the $\preceq_{\text {disp }}$-sense.

\section{Discussion}

In this paper, we have established necessary and sufficient conditions for dispersive inequalities between conditionals of bivariate distribution functions built from archimedean copulas, a phenomenon called dispersive cross-aging. Given the importance of the dispersive stochastic order relation in many applications, the results derived in this paper allow for a deeper understanding of the dependence structure induced by archimedean copulas. The conditions derived in this paper are easy to verify (at least numerically) and are satisfied by standard 
copulas including Clayton and Frank families for some marginal distributions (including Exponential and Pareto, as well as Gamma for appropriate values of Kendall's rank correlation coefficient).

The fact that comparisons in terms variability are not stable under non-decreasing transformations was expected since such transformations may affect the variability of the random variables. Hence, we cannot expect that the results derived for unit uniform marginals in Section 3 extend to arbitrary marginals. In other words, the results do not only depend on the underlying copula but the marginal distribution functions also matter. In the context of the present paper, the convexity of the transformation is an important property, as seen from Proposition 4.1. This stresses the difference between the approach developed in this paper and the standard analysis of dependence in which only the magnitude is taken into account (positive dependence generally means that large values of one component are accompanied by large values of the other). The present paper is a first step towards examining how the size of one component affects the variability of the other.

\section{Acknowledgements}

The Authors would like to express their gratitude to anonymous referees whose comments have been extremely useful to revise a previous version of the present work. The Authors also would like to thank Professors Esther Frostig and Christian Genest for interesting discussions on topics related to the contents of the present work. The financial support of the Onderzoeksfonds K.U. Leuven (GOA/07: Risk Modeling and Valuation of Insurance and Financial Cash Flows, with Applications to Pricing, Provisioning and Solvency) is gratefully acknowledged by Michel Denuit. Mhamed Mesfioui acknowledges the financial support of the Natural Sciences and Engineering Research Council of Canada.

\section{References}

- Belzunce, F., Candel,, J., and Ruiz, J.M. (1996). Dispersive orderings and characterizations of ageing classes. Statistics and Probability Letters 28, 321-327.

- Denuit, M., Dhaene, J., Goovaerts, M.J., and Kaas, R. (2005). Actuarial Theory for Dependent Risks: Measures, Orders and Models. Wiley, New York.

- Jeon, J., Kochar, S., and Park, C.G. (2006). Dispersive ordering - Some applications and examples. Statistical Papers 47, 227-247.

- Muller, A., and Scarsini, M. (2005). Archimedean copulae and positive dependence. Journal of Multivariate Analysis 93, 434-445.

- Nelsen, R.B. (2006). An Introduction to Copulas. Springer, New York.

- Pellerey, F., and Shaked, F. (1997). Characterizations of the IFR and DFR aging notions by means of the dispersive order. Statistics and Probability Letters 33, 389393 
- Shaked, M., and Shanthikumar, J.G. (2007). Stochastic Orders. Springer, New York. 